\section{Prevalência de sobrepeso em adolescentes: um estudo de base populacional em uma cidade no Sul do Brasil}

\author{
Prevalence of overweight in adolescents: \\ a population-based study in a southern \\ Brazilian city
}

Carmem L. Dutra 1

Cora L. Araújo 2

Andréa D. Bertoldi 1

\footnotetext{
1 Faculdade de Medicina, Universidade Federal de Pelotas, Pelotas, Brasil.

2 Faculdade de Nutrição, Universidade Federal de Pelotas, Pelotas, Brasil.

Correspondência C. L. Dutra

Programa de Pós-graduação em Epidemiologia, Faculdade de Medicina, Universidade Federal de Pelotas. Av. Duque de Caxias 250, 3 o piso, Pelotas, RS 96030-002, Brasil. clcdutra@hotmail.com
}

\section{Abstract}

The prevalence of overweight and its association with demographic, socioeconomic, behavioral, and biological variables were evaluated. The sample included 810 adolescents (10-19 years of age) living in the urban area of Pelotas, a southern Brazilian city. Overweight was defined as a body mass index $\geq$ the $85^{\text {th }}$ percentile, according to sex and age, and compared to the First National Health and Nutrition Examination Survey, following the WHO recommendation for adolescents. A clustering sampling strategy was used, and both the crude and adjusted analyses (Poisson Regression) took this strategy into account. The prevalence of overweight was 19.3\% (95\%CI: 16.6-22.0) and there was no difference between the sexes. The following groups presented a greater probability of being overweight: those classified in the wealthiest socioeconomic groups, those who had dieted to lose weight within the previous 3 months, those who watch 4 or more hours of television per day, and those who have less than 3 regular meals per day. After stratification by gender, high socioeconomic level was associated with greatest risk of overweight among boys. Dieting to lose weight during the previous 3 months, 4 or more hours of television viewing per day, and less than 3 formal meals per day were risk factors for overweight among girls.

Adolescent; Nutritional Assessment; Overweight

\section{Introdução}

O sobrepeso e a obesidade em crianças, adolescentes e adultos são problemas crescentes de saúde pública em países desenvolvidos. A Organização Mundial da Saúde (OMS) declarou recentemente ser a obesidade a nova síndrome mundial, tendo em vista que altas prevalências já podem ser encontradas também nas chamadas economias emergentes 1 .

A Pesquisa Nacional de Saúde e Nutrição (PNSN), realizada no Brasil em 1989, mostrou que $32,0 \%$ dos adultos apresentavam algum grau de excesso de peso, definido como Índice de Massa Corporal (IMC) $\geq 25 \mathrm{~kg} / \mathrm{m}^{2}$, sendo $27,0 \%$ na população masculina e $38,0 \%$ na feminina 2. A Região Sul apresentou a maior prevalência, tanto entre os homens $(34,0 \%)$, quanto entre as mulheres $(43,0 \%)$. Em Pelotas, Rio Grande do Sul, em dados relativos a 1994, a prevalência de obesidade (IMC $\geq 30 \mathrm{~kg} / \mathrm{m}^{2}$ ) em adultos com idades entre 20 e 69 anos foi de $21,0 \%$, sendo $25,0 \%$ entre as mulheres e $15,0 \%$ entre os homens, enquanto que quase $40,0 \%$ da amostra apresentaram sobrepeso (IMC entre 25 e $\left.29,9 \mathrm{~kg} / \mathrm{m}^{2}\right)^{3}$.

Dados do III National Health and Nutrition Examination Survey (NHANES III, 1988-1994) mostraram uma prevalência de sobrepeso de $10,6 \%$ em adolescentes norte-americanos entre 12 e 17 anos 4. Resultados da Pesquisa sobre Padrões de Vida (PPV) 5, realizada em 1997 nas 
regiões Sudeste e Nordeste do Brasil, mostraram que $10,4 \%$ dos adolescentes do sudeste entre 10 e 19 anos de idade apresentaram sobrepeso 5 . Estudo realizado em Pelotas em 1998, com adolescentes de 15-16 anos mostrou prevalência de sobrepeso de $20,2 \% 7$. Todos os estudos utilizaram o critério proposto pela OMS em 19956.

$\mathrm{O}$ aumento da obesidade também pode ser observado em crianças. A comparação de duas coortes de nascimento em Pelotas (1982 e 1993), mostrou que a prevalência de obesidade teve um aumento de 40,0\% no período de 11 anos, passando de $4,0 \%$ para $6,7 \%$, em crianças com 12 meses de idade 8 . A prevalência de sobrepeso nas crianças dessas mesmas coortes quando foram acompanhadas aos quatro anos de idade dobrou, sendo próxima de 5,0\% e 10,0\% em 1986 e 1997, respectivamente 9 .

A obesidade tem-se tornado um dos maiores problemas de saúde do século XXI, haja vista os agravos dela decorrentes e, conseqüentemente, do substancial aumento nos custos sociais de cuidados à saúde 10,11. Estudos têm mostrado que hipertensão arterial, doenças coronarianas, hiperlipoproteinemias, doenças osteoarticulares, diabetes mellitus e alguns tipos de câncer estão relacionados com a obesidade 12 .

Fatores ambientais e comportamentais têmse associado de forma complexa na determinação da obesidade. Sobrepeso e obesidade têmse relacionado de forma inconsistente com padrões sócio-econômicos, etnia e gênero não havendo um padrão definido de associação que seja generalizável. Em adultos, a prevalência de obesidade entre as mulheres de níveis de renda mais altos diminuiu, enquanto aumentou nas populações mais pobres e no sexo masculino 13. Alguns dos principais determinantes do sobrepeso e da obesidade, tais como práticas alimentares inadequadas, tempo que o adolescente permanece assistindo à televisão (TV), sobrepeso e obesidade nos pais, peso ao nascer e também as condições sócio-econômicas e ambientais são amplamente discutidos na literatura, na busca de compreender a gênese da obesidade, que é uma doença multicausal 14,15.

Uma vez que jovens com sobrepeso têm maior probabilidade de virem a se tornar adultos com sobrepeso e estes têm maiores riscos de adoecer, o sobrepeso na infância e na adolescência está ganhando reconhecimento como um importante problema de saúde pública 16,17.

Para melhor intervir no problema é fundamental conhecer sua magnitude assim como seus determinantes, a fim de buscar as melhores estratégias de prevenção. No Brasil, poucos são os estudos de base populacional que ava- liaram o sobrepeso e a obesidade em adolescentes de 10-19 anos. A maioria dos estudos brasileiros é de base escolar, portanto, não representativos da população. O presente estudo foi delineado com o objetivo de determinar a prevalência de sobrepeso em adolescentes da zona urbana do Município de Pelotas e estudar a sua associação com algumas variáveis demográficas, sócio-econômicas, comportamentais e biológicas.

\section{Metodologia}

No período de outubro a dezembro de 2003 realizou-se amplo inquérito sobre saúde, por meio de estudo transversal de base populacional, abrangendo crianças, adolescentes e adultos residentes na zona urbana da Cidade de Pelotas. O sobrepeso em adolescentes de 10-19 anos, de ambos os sexos, foi um dos tópicos estudados.

A amostra foi escolhida por meio de um processo por conglomerados, em múltiplos estágios. Para definição dos conglomerados foram utilizados os setores censitários do Censo Demográfico de 2000 (http:/ / www.ibge.gov.br, acessado em 01/Set/2003). Os 404 setores censitários do município foram ordenados conforme a renda média do chefe de família. Optouse por trabalhar com 144 setores censitários, com a finalidade de minimizar o efeito do delineamento amostral, visto que em estudos anteriores realizados em Pelotas encontrou-se efeitos de delineamento maiores com um número menor de setores censitários. Com base nas informações do Censo Demográfico de 2000 sobre o número de moradores por domicílio nas diversas faixas etárias, definiu-se que seria necessário estudar dez domicílios por setor. Os setores foram selecionados com probabilidade proporcional ao número de domicílios, ou seja, setores com maior número de domicílios tiveram maior chance de serem sorteados. Nos 144 setores sorteados realizou-se uma contagem de todos os domicílios, excluindo-se aqueles desabitados e unicamente comerciais. A seleção dos domicílios da amostra foi realizada sistematicamente de acordo com o número de domicílios do Censo Demográfico de 2000, dividido por dez. Porém, caso o número de domicílios do setor tivesse sido alterado desde o Censo Demográfico de 2000, o número de residências amostradas por setor poderia aumentar ou diminuir. O número total de domicílios sorteados foi de 1.530 , variando entre 8 e 27 por setor. Nos domicílios amostrados, os indivíduos entre 10 e 19 anos de idade foram elegíveis pa- 
ra o presente estudo. Foram excluídos os adolescentes institucionalizados (hospitais, prisões etc.), com incapacidade mental para responder o questionário, incapacidade física que impossibilitasse ou comprometesse as medidas antropométricas e gestantes.

O tamanho da amostra foi calculado visando a atender tanto o estudo de prevalência como o estudo de associação entre o sobrepeso e as variáveis independentes. Obteve-se uma amostra de 810 indivíduos, permitindo uma estimativa da prevalência de sobrepeso de $20,0 \%$ com margem de erro de $\pm 2,7$ pontos percentuais, com um nível de confiança de 95,0\%. Para o estudo da associação entre as variáveis independentes e sobrepeso pôde-se detectar riscos relativos iguais ou maiores que 2,0 , com $90,0 \%$ de poder para preditores com prevalências entre $15,0-85,0 \%$ e prevalência de sobrepeso de 18,0\% para os não-expostos, com nível de 5,0\% de significância.

Foram considerados com sobrepeso os adolescentes com IMC [peso (kg)/altura $\left(\mathrm{m}^{2}\right)$ ] por idade e sexo igual ou superior ao percentil 85, conforme a população de referência do $I \mathrm{Na}$ tional Health and Nutrition Examination Survey (NHANES I), de acordo com recomendação da OMS 6. As variáveis independentes foram: sexo, idade, cor da pele (branca/não-branca, segundo observação da entrevistadora), escolaridade (em anos completos de estudo), nível econômico (segundo classificação da Associação Brasileira de Empresas de Pesquisa - ABEP -, que leva em consideração a posse de bens de consumo, grau de instrução do chefe da família e presença de empregada mensalista, sendo a categoria "A" considerada como de melhor nível econômico (http://www.abep.org/codigosguias/ABEP_CCEB.pdf, acessado em 13/Set/ 2003), tipo de domicílio (casa ou apartamento), número de refeições diárias realizadas no dia anterior à entrevista (desjejum, colação, almoço, lanche da tarde, jantar e ceia), dieta para emagrecer nos últimos três meses, horas diárias assistindo à TV, tabagismo (sendo considerados fumantes aqueles que fumaram, no mínimo, um cigarro por dia nos últimos trinta dias), peso ao nascer (em gramas), presença de pêlos axilares, menarca nas meninas e mudança de voz nos meninos (as três últimas foram referidas pelos adolescentes). A escolaridade foi posteriormente agrupada em adequada ou não conforme a idade, sendo considerada inadequada quando na idade de dez anos o adolescente ainda não houvesse concluído a segunda série do ensino fundamental, com 11 anos a terceira série, e assim sucessivamente. As categorias extremas, A e E, da variável nível econômico apresentavam poucos indivíduos, por isso foram reagrupadas em A/B e D/E. Com relação ao tabagismo, o grupo dos ex-fumantes, por ser uma categoria pequena $(n=24)$, foi agrupado com os não-fumantes. O peso ao nascer foi categorizado em baixo peso $(<2.500 \mathrm{~g})$, peso normal e macrossômicos $(>4.000 \mathrm{~g})$.

$\mathrm{O}$ peso dos adolescentes foi medido com balanças portáteis, da marca Bathroom Scale, com capacidade para $150 \mathrm{~kg}$ e precisão de $1 \mathrm{~kg}$, calibradas e aferidas semanalmente com peso padrão. As entrevistadoras foram orientadas a registrar o peso conforme a última unidade completa em quilogramas, procedimento também adotado pelo Instituto Brasileiro de Geografia e Estatística na Pesquisa de Orçamentos Familiares (http://www.ibge.gov.br, acessado em 01/Mar/2004). A medida de altura foi obtida utilizando-se estadiômetros de alumínio portáteis, com precisão de um milímetro. As medidas antropométricas foram realizadas seguindo recomendação de Lohman et al. 18 .

Os dados foram coletados por entrevistadoras treinadas, usando-se um questionário padronizado e pré-codificado, previamente testado em estudo pré-piloto. Feitas as correções necessárias, realizou-se o estudo piloto em um setor censitário não-incluído na amostra do estudo a fim de testar a logística do trabalho de campo, treinar e selecionar as entrevistadoras.

Considerou-se perda ou recusa quando, após pelo menos três visitas da entrevistadora e uma do supervisor de campo, não foi possível realizar a entrevista. Foram revisitados 10,0\% dos entrevistados, selecionados aleatoriamente por setor censitário, com aplicação de um questionário reduzido contendo questões-chave para verificação das respostas. A concordância entre as informações coletadas no estudo e no controle de qualidade foi avaliada através do cálculo do índice kappa, 19 utilizando-se a pergunta referente à dieta nos três meses que precederam a entrevista. O valor kappa para a questão escolhida mostrou um coeficiente de concordância de 0,83 , correspondendo a um nível de classificação excelente. O tempo máximo decorrido entre a entrevista e a revisita para o controle de qualidade foi de 14 dias. Além disso, foram feitas ligações telefônicas para cerca de $10,0 \%$ dos entrevistados a fim de verificar especificamente a realização das medidas antropométricas.

Os questionários, após codificados e revisados, foram duplamente digitados utilizando-se o programa Epi Info versão 6.04, com checagem automática de consistência e amplitude. A análise dos dados foi realizada com o programa Stata 8.0, levando em consideração a estratégia amostral por conglomerados. 
A análise das associações foi feita no total dos indivíduos e estratificada por sexo, por entender-se que os determinantes do sobrepeso diferem entre meninos e meninas. Nas análises utilizou-se os testes de Wald para comparação de proporções e para avaliar tendência linear. Para a estimativa ajustada de razões de prevalência, utilizou-se a regressão de Poisson 20. A análise multivariável seguiu um modelo hierárquico 21 , sendo que no nível mais distal de determinação encontram-se as variáveis demográficas (sexo, idade e cor da pele) e sócioeconômicas (escolaridade e nível econômico), e no nível mais proximal as variáveis dieta para emagrecer nos últimos três meses, horas diárias de TV e número de refeições diárias. Foram realizados ajustes para as variáveis do mesmo nível e nível superior que apresentaram um valor $\mathrm{p} \leq 0,2$ na análise ajustada, evitando-se dessa forma, ajustes para fatores mediadores.

O presente estudo foi aprovado pela Comissão de Ética e Pesquisa da Faculdade de Medicina, Universidade Federal de Pelotas. Os dados foram coletados após consentimento informado dos entrevistados, sendo-lhes assegurado o sigilo sobre as informações individuais.

\section{Resultados}

Nos 1.530 domicílios sorteados foram encontrados 826 adolescentes elegíveis para o estudo. Houve $1,9 \%(n=16)$ de perdas ou recusas, totalizando 810 adolescentes entrevistados. Entre os não-respondentes, nove eram meninas e sete meninos.

A prevalência de sobrepeso foi de $19,3 \%$ (IC95\%: 16,6-22,0), com um efeito de delineamento de 1,23 e correlação intraclasse de 0,062. Não houve diferença na prevalência de sobrepeso entre meninos e meninas. Considerandose o critério proposto por Cole et al. 22 , a prevalência de sobrepeso foi de $21,8 \%$ e a de obesidade de $4,5 \%$. Na Tabela 1 estão descritas as variáveis demográficas, sócio-econômicas, comportamentais e biológicas. A média de idade dos adolescentes estudados foi de 14,7 anos (DP $\pm 2,9)$, sendo 50,3\% $(n=407)$ meninos. A amostra constituiu-se, principalmente, por brancos $(73,2 \%)$, com escolaridade adequada (63,6\%) e dos níveis econômicos D e E (46,3\%).

Em relação ao tipo de domicílio, $84,4 \%$ dos adolescentes moravam em casa. Dois terços dos adolescentes entrevistados realizaram três ou quatro refeições no dia anterior à entrevista. A média do número de refeições foi de 3,8 (DP $\pm 1,1)$. Aproximadamente 9,0\% realizaram dieta para emagrecer nos três meses que prece- deram a entrevista $(14,4 \%$ das meninas e $3,0 \%$ dos meninos). A média de horas diárias de TV foi de 3,5 horas (DP $\pm 2,6$ ) e quase $30,0 \%$ da amostra costumavam assistir à televisão por quatro horas ou mais durante o dia. Quanto ao tabagismo, 8,0\% dos adolescentes eram fumantes.

Quase 9,0\% dos adolescentes estudados nasceram com baixo peso. Entre os meninos, 13,4\% nasceram com peso igual ou superior a $4.000 \mathrm{~g}$, sendo que nas meninas a freqüência de macrossomia foi de $6,2 \%$ (Tabela 1). É importante ressaltar que para $16,5 \%$ dos adolescentes não foi possível obter informação sobre o peso ao nascer e entre os 676 com informação, 20,4\% referiram um valor de peso aproximado. $\mathrm{O}$ peso ao nascer do adolescente foi informado em $75,5 \%$ dos casos pelas mães, $16,7 \%$ pelo próprio adolescente e $7,8 \%$ por outros familiares.

A Tabela 2 apresenta as prevalências e razões de prevalências brutas. Não houve diferença na ocorrência de sobrepeso entre os sexos e também em relação à idade e à cor da pele. A escolaridade e o nível econômico estiveram associados com o sobrepeso na amostra total e nos meninos. Com relação ao tipo de domicílio não se verificou diferença nas prevalências de sobrepeso para os adolescentes que moram em casa ou em apartamento $(\mathrm{p}=0,8)$ (Tabela 2).

O número de refeições diárias mostrou-se inversamente associado ao sobrepeso em ambos os sexos. Ter realizado dieta para emagrecer nos três meses que antecederam a entrevista também apresentou associação significativa com o sobrepeso para a amotra total e em ambos os sexos. O número de horas diárias de TV esteve diretamente associado com sobrepeso, mas não mostrou associação estatisticamente significativa para os meninos.

Nos fumantes, a prevalência de sobrepeso foi menor do que entre os não-fumantes. A diferença na prevalência de sobrepeso entre fumantes e não-fumantes foi maior nos meninos do que nas meninas. Essas diferenças, entretanto, não foram estisticamente significativas (Tabela 2).

A prevalência de sobrepeso foi diretamente proporcional ao peso ao nascer, porém as diferenças encontradas não foram estatisticamente significativas (Tabela 2).

A Tabela 3 apresenta a análise ajustada para potenciais fatores de confusão segundo o modelo hierárquico de análise descrito na metodologia. Quanto ao sexo não houve diferença na ocorrência de sobrepeso. O mesmo aconteceu em relação à idade, cor da pele e escolaridade. O nível econômico esteve associado com o sobrepeso na amostra total e nos meninos, sendo que a freqüência de sobrepeso foi maior 
Tabela 1

Descrição da amostra conforme variáveis demográficas, sócio-econômicas, comportamentais e biológicas.

Pelotas, Rio Grande do Sul, Brasil, 2003 ( $N=810)$.

\begin{tabular}{|c|c|c|c|c|c|c|}
\hline \multirow[t]{2}{*}{ Variáveis* } & \multicolumn{2}{|c|}{ Todos } & \multicolumn{2}{|c|}{ Meninos } & \multicolumn{2}{|c|}{ Meninas } \\
\hline & $\mathrm{n}$ & $\%$ & $n$ & $\%$ & $\mathrm{n}$ & $\%$ \\
\hline \multicolumn{7}{|l|}{ Idade (anos) } \\
\hline $10-14$ & 378 & 46,7 & 200 & 49,1 & 178 & 44,2 \\
\hline $15-19$ & 432 & 53,3 & 207 & 50,9 & 225 & 55,8 \\
\hline \multicolumn{7}{|l|}{ Cor da pele } \\
\hline Branca & 593 & 73,2 & 299 & 73,5 & 294 & 73,0 \\
\hline Não-branca & 217 & 26,8 & 108 & 26,5 & 109 & 27,0 \\
\hline \multicolumn{7}{|l|}{ Escolaridade } \\
\hline Inadequada & 291 & 36,4 & 155 & 38,8 & 136 & 34,0 \\
\hline Adequada & 508 & 63,6 & 244 & 61,2 & 264 & 66,0 \\
\hline \multicolumn{7}{|c|}{ Nível econômico } \\
\hline A/B & 188 & 23,4 & 96 & 23,8 & 92 & 22,9 \\
\hline$c$ & 244 & 30,3 & 126 & 31,2 & 118 & 29,4 \\
\hline $\mathrm{D} / \mathrm{E}$ & 373 & 46,3 & 182 & 45,0 & 191 & 47,7 \\
\hline \multicolumn{7}{|c|}{ Tipo de domicílio } \\
\hline Casa & 684 & 84,4 & 349 & 85,8 & 335 & 83,1 \\
\hline Apartamento & 126 & 15,6 & 58 & 14,2 & 68 & 16,9 \\
\hline \multicolumn{7}{|c|}{ Refeições diárias } \\
\hline $1-2$ & 84 & 10,4 & 30 & 7,4 & 54 & 13,4 \\
\hline 3 & 238 & 29,4 & 111 & 27,3 & 127 & 31,5 \\
\hline 4 & 303 & 37,4 & 159 & 39,0 & 144 & 35,7 \\
\hline $5-6$ & 185 & 22,8 & 107 & 26,3 & 78 & 19,4 \\
\hline \multicolumn{7}{|c|}{ Dieta para emagrecer } \\
\hline Não & 740 & 91,4 & 395 & 97,0 & 345 & 85,6 \\
\hline Sim & 70 & 8,6 & 12 & 3,0 & 58 & 14,4 \\
\hline \multicolumn{7}{|c|}{ Horas diárias de TV } \\
\hline$<2$ & 199 & 24,6 & 106 & 26,1 & 93 & 23,1 \\
\hline $2-3,9$ & 376 & 46,4 & 193 & 47,4 & 183 & 45,4 \\
\hline 4 ou mais & 235 & 29,0 & 108 & 26,5 & 127 & 31,5 \\
\hline \multicolumn{7}{|l|}{ Tabagismo } \\
\hline Não-fumante & 745 & 92,0 & 380 & 93,4 & 365 & 90,6 \\
\hline Fumante & 65 & 8,0 & 27 & 6,6 & 38 & 9,4 \\
\hline \multicolumn{7}{|c|}{ Peso ao nascer (g) } \\
\hline$<2.500$ & 60 & 8,9 & 27 & 8,1 & 33 & 9,7 \\
\hline $2.500-3.999$ & 550 & 81,3 & 263 & 78,5 & 287 & 84,1 \\
\hline$>4.000$ & 66 & 9,8 & 45 & 13,4 & 21 & 6,2 \\
\hline
\end{tabular}

* O número máximo de valores ignorados foi de 134 para a variável peso ao nascer.

Nas demais variáveis os valores ignorados foram inferiores a 11. 
Tabela 2

Prevalências e razões de prevalências brutas para sobrepeso em adolescentes segundo variáveis demográficas,

sócio-econômicas, comportamentais e biológicas. Pelotas, Rio Grande do Sul, Brasil, 2003.

\begin{tabular}{|c|c|c|c|c|c|c|c|c|c|}
\hline Variáveis & $\%$ & $\begin{array}{c}\text { Geral } \\
\text { RP bruta (IC95\%) }\end{array}$ & Valor-p* & $\%$ & $\begin{array}{c}\text { Meninos } \\
\text { RP bruta (IC95\%) }\end{array}$ & Valor-p* & $\%$ & $\begin{array}{c}\text { Meninas } \\
\text { RP bruta (IC95\%) }\end{array}$ & Valor-p* \\
\hline Sobrepeso** & 19,3 & - & - & 19,6 & - & - & 19,1 & - & - \\
\hline Idade (anos) & & & $0,09 *$ & & & $0,1^{*}$ & & & $0,4^{*}$ \\
\hline $10-14$ & 22,1 & $1,32(0,96-1,81)$ & & 23,1 & $1,44(0,93-2,21)$ & & 21,0 & $1,20(0,79-1,84)$ & \\
\hline $15-19$ & 16,8 & 1,00 & & 16,1 & 1,00 & & 17,5 & 1,00 & \\
\hline Cor da pele & & & $0,8^{*}$ & & & $0,4^{\star}$ & & & $0,7^{\star}$ \\
\hline Branca & 19,6 & $1,05(0,75-1,46)$ & & 20,5 & $1,21(0,77-1,89)$ & & 18,6 & $0,92(0,57-1,49)$ & \\
\hline Não-branca & 18,6 & 1,00 & & 17,0 & 1,00 & & 20,2 & 1,00 & \\
\hline Escolaridade & & & $0,04^{\star}$ & & & $0,03^{*}$ & & & $0,4^{*}$ \\
\hline Inadequada & 15,3 & 1,00 & & 14,4 & 1,00 & & 16,3 & 1,00 & \\
\hline Adequada & 21,4 & $1,40(1,02-1,92)$ & & 23,0 & $1,60(1,06-2,41)$ & & 19,9 & $1,22(0,75-1,98)$ & \\
\hline Nível econômico & & & $0,003^{*}$ & & & $<0,001^{\star}$ & & & $0,3^{*}$ \\
\hline $\mathrm{A} / \mathrm{B}$ & 28,3 & $1,63(1,16-2,29)$ & & 34,4 & $2,56(1,59-4,15)$ & & 22,0 & $1,04(0,64-1,69)$ & \\
\hline C & 15,6 & $0,90(0,58-1,39)$ & & 17,5 & $1,30(0,71-2,39)$ & & 13,7 & $0,65(0,35-1,19)$ & \\
\hline$D / E$ & 17,4 & 1,00 & & 13,4 & 1,00 & & 21,2 & 1,00 & \\
\hline Tipo de domicílio & & & $0,8^{*}$ & & & $0,2^{*}$ & & & $0,08^{*}$ \\
\hline Casa & 19,5 & $1,06(0,73-1,53)$ & & 18,5 & $0,72(0,45-1,15)$ & & 20,5 & $1,72(0,93-3,17)$ & \\
\hline Apartamento & 18,4 & 1,00 & & 25,9 & 1,00 & & 11,9 & 1,00 & \\
\hline Refeições diárias & & & $<0,001^{\star \star \star}$ & & & $0,02^{\star \star \star}$ & & & $<0,001^{\star * *}$ \\
\hline $1-2$ & 30,1 & $2,62(1,55-4,43)$ & & 23,3 & $1,88(0,83-4,28)$ & & 34,0 & $3,31(1,49-7,37)$ & \\
\hline 3 & 23,6 & $2,06(1,35-3,14)$ & & 25,2 & $2,04(1,15-3,60)$ & & 22,2 & $2,17(1,04-4,50)$ & \\
\hline 4 & 17,7 & $1,54(0,99-2,40)$ & & 19,6 & $1,58(0,92-2,72)$ & & 15,5 & $1,51(0,69-3,33)$ & \\
\hline $5-6$ & 11,5 & 1,00 & & 12,4 & 1,00 & & 10,3 & 1,00 & \\
\hline Dieta para emagrecer & & & $0,002^{*}$ & & & 0,03 * & & & $0,008 *$ \\
\hline Não & 17,9 & 1,00 & & 18,9 & 1,00 & & 16,9 & 1,00 & \\
\hline Sim & 34,3 & $1,91(1,27-2,89)$ & & 41,7 & $2,21(1,08-4,50)$ & & 32,7 & $1,94(1,19-3,16)$ & \\
\hline Horas diárias de TV & & & $0,008^{\star \star \star}$ & & & $0,2^{\star \star \star}$ & & & $0,006^{\star \star *}$ \\
\hline$<2$ & 14,7 & 1,00 & & 17,1 & 1,00 & & 12,0 & 1,00 & \\
\hline $2-3,9$ & 17,6 & $1,20(0,80-1,78)$ & & 17,6 & $1,03(0,61-1,73)$ & & 17,6 & $1,47(0,82-2,64)$ & \\
\hline 4 ou mais & 26,0 & $1,76(1,14-2,73)$ & & 25,5 & $1,49(0,82-2,70)$ & & 26,4 & $2,21(1,23-3,96)$ & \\
\hline Tabagismo & & & $0,4^{\star}$ & & & $0,3^{*}$ & & & $0,7^{*}$ \\
\hline Não-fumante & 19,7 & $1,38(0,68-2,79)$ & & 20,1 & $1,74(0,60-5,04)$ & & 19,3 & $1,19(0,52-2,74)$ & \\
\hline Fumante & 14,3 & 1,00 & & 11,5 & 1,00 & & 16,2 & 1,00 & \\
\hline Peso ao nascer $(\mathrm{g})$ & & & $0,06^{\star \star \star}$ & & & $0,09 * \star \star$ & & & $0,4^{\star \star \star}$ \\
\hline$<2.500$ & 11,9 & 1,00 & & 7,7 & 1,00 & & 15,2 & 1,00 & \\
\hline $2.500-3.999$ & 20,7 & $1,74(0,85-3,58)$ & & 20,6 & $2,68(0,70-10,32)$ & & 20,8 & $1,37(0,59-3,20)$ & \\
\hline$>4.000$ & 25,8 & $2,17(0,92-5,13)$ & & 26,7 & $3,47(0,77-5,58)$ & & 23,8 & $1,57(0,54-4,56)$ & \\
\hline
\end{tabular}

* Teste de Wald para heterogeneidade de proporções;

** A RP e IC95\% para a variável sexo foram de 0,97 $(0,73-1,29)$ e o valor-p $=0,9$;

$\star \star \star$ Teste de Wald para tendência linear. 
Análise ajustada para as associações de variáveis demográficas, sócio-econômicas e comportamentais seguindo um modelo de análise hierarquizado. Pelotas, Rio Grande do Sul, Brasil, 2003.

\begin{tabular}{|c|c|c|c|c|}
\hline Nível* & Variáveis & $\begin{array}{c}\text { Geral } \\
\text { Valor-p** } \\
\operatorname{RP}(I C 95 \%)\end{array}$ & $\begin{array}{c}\text { Meninos } \\
\text { Valor-p** } \\
\text { RP (IC95\%) }\end{array}$ & $\begin{array}{c}\text { Meninas } \\
\text { Valor-p** } \\
\operatorname{RP}(I C 95 \%)\end{array}$ \\
\hline \multirow[t]{3}{*}{1} & Idade (anos) & $0,06 \#$ & $0,07 \#$ & $0,5^{\prime}$ \\
\hline & 10-14 & $1,35(0,98-1,85)$ & $1,47(0,97-2,22)$ & $1,15(0,73-1,80)$ \\
\hline & $15-19$ & 1,00 & 1,00 & 1,00 \\
\hline \multirow[t]{3}{*}{1} & Cor da pele & $0,6 \#$ & $0,7 \#$ & $1,0 \#$ \\
\hline & Branca & $0,92(0,65-1,30)$ & $0,90(0,58-1,42)$ & $1,00(0,60-1,68)$ \\
\hline & Não-branca & 1,00 & 1,00 & 1,00 \\
\hline \multirow[t]{3}{*}{1} & Escolaridade & $0,09 \#$ & $0,8 \#$ & $0,3 \#$ \\
\hline & Inadequada & 1,00 & 1,00 & 1,00 \\
\hline & Adequada & $1,16(0,80-1,66)$ & $1,07(0,67-1,69)$ & $1,37(0,78-2,41)$ \\
\hline \multirow[t]{4}{*}{1} & Nível econômico & $0,002 \#$ & $0,0003 \#$ & $0,3 \#$ \\
\hline & $\mathrm{A} / \mathrm{B}$ & $1,66(1,18-2,32)$ & $2,60(1,62-4,17)$ & $1,04(0,64-1,69)$ \\
\hline & C & $0,90(0,58-1,40)$ & $1,34(0,73-2,46)$ & $0,65(0,35-1,19)$ \\
\hline & $D / E$ & 1,00 & 1,00 & 1,00 \\
\hline \multirow[t]{5}{*}{2} & Refeições diárias & $<0,001 \# \#$ & $0,07 \# \#$ & $0,002 \# \#$ \\
\hline & $1-2$ & $2,40(1,38-4,19)$ & $1,53(0,67-3,49)$ & $2,95(1,29-6,74)$ \\
\hline & 3 & $1,98(1,29-3,04)$ & $1,87(1,05-3,34)$ & $1,88(0,90-3,91)$ \\
\hline & 4 & $1,41(0,91-2,20)$ & $1,41(0,82-2,45)$ & $1,35(0,61-2,97)$ \\
\hline & $5-6$ & 1,00 & 1,00 & 1,00 \\
\hline \multirow[t]{3}{*}{2} & Dieta para emagrecer & $0,03 \#$ & $0,1 \#$ & $0,04 \#$ \\
\hline & Não & 1,00 & 1,00 & 1,00 \\
\hline & Sim & $1,59(1,04-2,44)$ & $1,63(0,87-3,03)$ & $1,75(1,04-2,93)$ \\
\hline \multirow[t]{4}{*}{2} & Horas diárias de TV & $0,02 \# \#$ & $0,4 \# \#$ & $0,007 \# \#$ \\
\hline & $<2$ & 1,00 & 1,00 & 1,00 \\
\hline & $2-3,9$ & $1,14(0,76-1,69)$ & $0,87(0,50-1,53)$ & $1,56(0,89-2,74)$ \\
\hline & 4 ou mais & $1,61(1,05-2,46)$ & $1,22(0,66-2,27)$ & $2,16(1,23-3,80)$ \\
\hline
\end{tabular}

Nota: A RP e IC95\% para a variável sexo foram de 0,96 $(0,72-1,28)$ e o valor-p\# = 0,8, ajustado para todas as variáveis do primeiro nível;

* Todas as variáveis estão controladas para as demais do mesmo nível e para as do nível acima;

** As variáveis com nível de significância $\leq 20,0 \%$ foram mantidas no modelo para controle de confusão;

\# Teste de Wald para heterogeneidade de proporções;

\#\# Teste de Wald para tendência linear.

nos adolescentes de nível econômico mais alto. Nas meninas não foi encontrada essa associação.

O número de refeições diárias esteve inversamente associado ao sobrepeso, ou seja, quanto menor o número de refeições realizadas, maior a freqüência de sobrepeso. Este efeito foi maior nas meninas do que nos meninos (Tabela 3).

$\mathrm{Na}$ amostra total o sobrepeso foi $59,0 \%$ maior naqueles adolescentes que referiram realizar dieta para emagrecer nos últimos três meses quando comparados àqueles que não fizeram; nas meninas o sobrepeso foi $75,0 \%$ maior naquelas que realizavam dieta. Entre os meninos, a dieta para emagrecer realizada nos três meses que precederam a entrevista perdeu significância estatística quando ajustada para idade, nível econômico e número de refeições diárias (Tabela 3).

A freqüência de sobrepeso foi maior conforme aumentou o número diário de horas de $\mathrm{TV}$, sendo mais elevada nas meninas quando comparadas à amostra total. Entre os meninos não se encontrou tal associação (Tabela 3). 
A seguir estão apresentados resultados de análises bivariadas entre algumas variáveis independentes, apenas com o objetivo de explorar possíveis associações que possibilitariam um melhor entendimento da associação entre estas e o sobrepeso. Esses resultados não estão apresentados em tabelas.

Ao comparar a escolaridade dos adolescentes segundo as categorias econômicas, observou-se que quase $73,0 \%$ daqueles com escolaridade inadequada pertenciam aos níveis econômicos $\mathrm{D} / \mathrm{E}$, enquanto que somente $5,9 \%$ pertenciam aos níveis $\mathrm{A} / \mathrm{B}$.

$\mathrm{Na}$ análise estratificada entre tipo de domicílio conforme idade e sexo, verificou-se que os meninos de 10-14 anos, residentes em apartamentos, apresentaram mais do que o dobro de risco para sobrepeso $(45,8 \%)$ comparativamente aos meninos de 10-14 anos que moravam em casa $(20,0 \%)(p=0,005)$. O mesmo não se observou para as meninas de 10-14 anos e para os adolescentes de 15-19 anos, de ambos os sexos.

Com relação ao número de horas diárias de TV segundo a idade verificou-se que $35,2 \%$ dos adolescentes entre 10 e 14 anos e $23,6 \%$ daqueles entre 15 e 19 anos assistiam quatro horas ou mais de TV durante o dia ( $\mathrm{p}<0,001)$.

Quanto à maturação sexual, 74,0\% dos adolescentes referiram já ter pêlos axilares, sendo $63,9 \%$ dos meninos e $83,4 \%$ das meninas. A prevalência de sobrepeso nos adolescentes que referiram já ter pêlos axilares foi de 19,8\% e de $17,8 \%$ nos que referiram não tê-los (sem diferença por sexo). A diferença de sobrepeso encontrada entre os adolescentes que possuíam ou não pêlos axilares não foi estatisticamente significativa.

Entre as meninas, $77,7 \%(n=313)$ referiram já ter tido a menarca. A idade média de ocorrência da menarca foi de 12,2 anos (DP $\pm 1,2$ ). A prevalência de sobrepeso nas meninas que tiveram a menarca foi de $20,7 \%$ e de $13,3 \%$ naquelas que ainda não haviam tido $(\mathrm{p}=0,1)$.

Nos meninos, 34,9\% $(n=142)$ relataram já ter tido a mudança de voz, $45,9 \%(n=187)$ afirmaram estar na fase de transição da voz infantil para a de adulto e $19,2 \%(n=78)$ referiram que sua voz não havia mudado. A prevalência de sobrepeso nos meninos que informaram já ter tido a mudança de voz foi de $17,6 \%$, entre aqueles que relataram estar com a voz em transição foi de $21,1 \%$ e de $19,5 \%$ naqueles que a voz não havia mudado $(\mathrm{p}=0,8)$.

\section{Discussão}

O presente estudo foi realizado com uma amostra representativa dos adolescentes da zona urbana do Município de Pelotas. O baixo percentual de perdas e recusas minimiza a possibilidade de viés de seleção.

Utilizou-se um delineamento transversal, que apresenta vantagens quanto ao custo e tempo de coleta de dados. Entretanto, sendo o desfecho e as exposições coletadas num único momento, os achados podem ser afetados pelo viés de causalidade reversa, ou seja, a exposição estudada pode ser influenciada pela ocorrência do desfecho. No presente estudo devese considerar a possibilidade de que a ocorrência de sobrepeso poderia modificar o comportamento de algumas variáveis explanatórias, tais como: número de refeições diárias, dieta para emagrecer nos últimos três meses, hábito de assistir à televisão e tabagismo. Em vista disso, as associações identificadas não devem ser interpretadas como relações causais. Também pode ter ocorrido erro recordatório, bem como um viés de memória, na coleta do peso ao nascer. Baixo peso ou macrossomia podem ter sido recordados com maior precisão do que pesos considerados normais. A categorização dessa variável buscou minimizar a influência desse viés.

Em estudos transversais com desfechos dicotômicos utiliza-se freqüentemente a regressão logística, cujo resultado é expresso em razão de “odds". Quando o desfecho apresenta freqüência elevada a razão de "odds" superestima a razão de prevalência. Em vista disso, optou-se pela regressão de Poisson que estima diretamente a razão de prevalência, facilitando a interpretação dos resultados 20.

A prevalência de sobrepeso encontrada entre os adolescentes foi elevada. Dados da Pesquisa sobre Padrões de Vida (PPV-1997), também analisados segundo o critério proposto pela OMS 6, mostraram prevalências de 9,3\% para as meninas e de $7,3 \%$ para meninos 5 . Diferenças na definição de sobrepeso e faixas etárias dificultaram a comparação entre esse e outros estudos. Considerou-se altas as prevalências de sobrepeso encontradas neste estudo pois crianças e adolescentes obesos têm uma maior chance de virem a ser adultos obesos 4,17.

No presente estudo não foram encontradas diferenças significativas nas prevalências de sobrepeso entre os sexos, similarmente ao que foi encontrado no estudo de Ogden et al. 23. Já os resultados da PNSN-1989 mostram que as prevalências de sobrepeso em adolescentes de 10-19 anos, segundo o critério definido pela 
OMS 6 , foi de $10,6 \%$ entre as meninas e de $4,8 \%$ entre os meninos 24 .

Embora a prevalência de sobrepeso tenha sido maior nos adolescentes de 10-14 anos, esta diferença não foi estatisticamente significativa. No estudo de Veiga et al. 25 com adolescentes residentes em região urbana do Sudeste do Brasil, onde as idades foram categorizadas de 10-13, 14-16 e 17-19 anos, as prevalências de sobrepeso analisadas segundo Cole et al. 22 também foram maiores nos adolescentes mais novos, em ambos os sexos.

No presente estudo não foram encontradas diferenças de sobrepeso segundo a cor da pele, entretanto, estudos norte-americanos mostram maior prevalência de sobrepeso em adolescentes não-brancos 23,26. Existe consistente relação entre grupo étnico e sobrepeso, sendo que a raça negra parece ser mais exposta ao desenvolvimento da obesidade e suas co-morbidades. Isto deve-se a fatores biológicos, como a concentração de lipólise corporal total, diferentemente do que ocorre no grupo étnico branco, onde o fator biológico não é o principal predisponente, mas provavelmente os fatores ambientais que podem tanto aumentar a prevalência em função de maior disponibilidade de alimentos, quanto diminuí-la em função de maior acesso à informação e a serviços de saúde 27 .

Encontrou-se prevalência maior de sobrepeso em adolescentes com escolaridade adequada em relação à idade. Resultado similar foi encontrado em estudo que analisou dados da PNSN 24. Indivíduos com escolaridade adequada são aqueles com melhor condição socioeconômica e conseqüentemente com maior acesso aos alimentos, o que poderia explicar a maior ocorrência de sobrepeso nesse grupo.

Os resultados deste estudo estão de acordo com outros realizados no Brasil com crianças e adolescentes, os quais mostram que a renda está diretamente associada ao sobrepeso 13,24,28. Entretanto, estudos realizados na China (1997) e na Rússia (1998) não encontraram diferenças nas prevalências de sobrepeso segundo tercis de renda per capita. Já nos Estados Unidos, os dados do NHANES III (1988-1994) mostram prevalência de sobrepeso maior no tercil de renda mais baixa 28 .

Com relação ao tipo de domicílio, observou-se que apenas os meninos de 10-14 anos que moram em apartamentos apresentaram maior risco para sobrepeso. Isto pode dever-se ao fato de que os meninos mais jovens residentes em apartamentos têm menor possibilidade de serem ativos enquanto que os demais, por serem mais velhos, poderiam realizar atividades físicas fora de casa com maior facilidade.
Maiores prevalências de sobrepeso foram encontradas entre os adolescentes que realizavam menor número de refeições diárias. Esta associação manteve-se após ajuste para idade, escolaridade, nível econômico, dieta para emagrecer e número de horas diárias de TV. Suprimir refeições pode ser uma estratégia de emagrecimento adotada pelos adolescentes na tentativa de perder peso. Outra possibilidade, seria a de que adolescentes portadores de sobrepeso poderiam estar subestimando o número de refeições diárias. Além disso, a associação entre sobrepeso e número de refeições diárias deve ser interpretada com cautela devido à possibilidade de causalidade reversa.

Conforme esperado, a prevalência de sobrepeso foi maior naqueles adolescentes que referiram ter realizado dieta para emagrecer nos últimos três meses. Resultado semelhante foi encontrado por Fonseca et al. 29. Estudo realizado na Suíça mostrou que já com idades entre 6-12 anos, $19,0 \%$ dos meninos e $34,0 \%$ das meninas estavam tentando perder peso 30 . Esses achados foram muito superiores aos encontrados no presente estudo com relação à dieta para emagrecer, o que pode refletir uma alta prevalência de sobrepeso no estudo suíço $(34,0 \%)$ ou também a busca de padrões estéticos de magreza.

A associação encontrada no presente estudo entre número de horas diárias que os adolescentes permanecem assistindo à TV e sobrepeso está de acordo com os achados de outros estudos 29,31,32,33. Estudo transversal realizado em Niterói, Rio de Janeiro, com adolescentes de 15-17 anos, mostrou prevalência de sobrepeso duas vezes maior nos meninos que assistiam à TV por três horas ou mais, comparado com o grupo que assistia por, no máximo, uma hora diária 29. Tal associação talvez possa ser explicada por ser essa uma atividade sedentária agravada pela maior oportunidade de consumo de alimentos, geralmente hipercalóricos. Além disso, estariam mais expostos à propaganda de alimentos considerados não-saudáveis quando consumidos em excesso. Como não há relação temporal entre a exposição e o desfecho, essa associação não deve ser interpretada como sendo causal.

A prevalência de sobrepeso no presente estudo foi maior nos adolescentes não-fumantes. A relação entre tabagismo e obesidade em adolescentes tem sido pouco estudada, o que dificultou a comparação dos resultados do presente estudo com a literatura 34 . Estudo transversal realizado em Pelotas com adolescentes de 10-19 anos encontrou prevalência de tabagismo de $12,1 \% 35$, valor esse um pouco superior ao encontrado neste estudo. 
As prevalências de sobrepeso foram maiores conforme aumentava o peso ao nascer. Diversos estudos têm descrito o peso ao nascer como preditor da obesidade em adolescentes e adultos 15,17,36. Parsons et al. 15 estudaram a relação entre peso ao nascer e IMC em adultos, por meio de uma revisão sistemática de oito estudos, dentre os quais, cinco apresentaram relação positiva entre peso ao nascer e IMC em adultos. Entretanto, outros determinantes muito precoces tais como o crescimento intra-uterino e obesidade materna têm-se mostrado de risco para sobrepeso na adolescência e podem confundir associações mais proximais como o peso ao nascer 14 .

As maiores prevalências de sobrepeso encontradas nas meninas que já tiveram a menarca, e menores nos meninos que referiram já ter mudado a voz estão de acordo com os achados na literatura 17,24 . Semelhante resultado foi encontrado por Neutzling et al. 24 com relação à menarca nas meninas. Laitinen et al. $17 \mathrm{ob}$ servaram em coorte de nascidos em 1966 no norte da Finlândia que quanto mais cedo era a idade da menarca, maior o sobrepeso em adultos aos 31 anos de idade. Com relação ao estágio puberal dos adolescentes, deve-se considerar que a menarca é uma medida com alta validade e repetibilidade, e a voz de adulto possui repetibilidade e validade média 6 .

Conclui-se que a prevalência de sobrepeso encontrada no presente estudo foi alta. A maioria das associações mostrou-se diferente entre meninos e meninas. Os resultados revelaram prevalências maiores de sobrepeso nos adolescentes de nível econômico mais elevado, naqueles que realizavam dieta para emagrecer, nos que costumavam fazer menos de três refeições diárias e entre aqueles que permaneciam diariamente assistindo à TV por quatro horas ou mais.

Com base nesses resultados, o sobrepeso já deve ser entendido como um problema emergente. Para enfrentá-lo, intervenções visando às mudanças no estilo de vida, tais como hábitos alimentares saudáveis e prática de atividade física podem ser mais efetivas quando dirigidas aos estágios mais precoces de seu desenvolvimento. Além de ações individuais é fundamental que as políticas de alimentação e nutrição disponibilizem cuidados nas várias esferas em que o adolescente se insere. Nas escolas, atenção à qualidade da merenda escolar e dos alimentos oferecidos nas cantinas, além da educação nutricional e da promoção à atividade física devem ser os focos das medidas preventivas e de tratamento. No ambiente familiar, os pais devem dar exemplo de um padrão alimentar saudável, bem como estimular a atividade física, orientando para a redução do número de horas que as crianças e adolescentes assistem à TV, jogam videogame ou permanecem na frente do computador. Alertar mães, jovens e adultos sobre as conseqüências da obesidade, bem como orientar com relação a hábitos alimentares saudáveis e a importância da realização da atividade física são importantes medidas preventivas, em todas as camadas sociais.

\section{Resumo}

Avaliou-se a prevalência de sobrepeso em adolescentes e sua distribuição segundo varíaveis demográficas, sócio-econômicas, comportamentais e biológicas. Foram entrevistados 810 adolescentes entre 10 e 19 anos, residentes na zona urbana de Pelotas, Rio Grande do Sul, Brasil. O sobrepeso foi definido de acordo com a OMS (IMC $\geq$ Percentil 85, segundo idade e sexo conforme a população de referência do I National Health and $\mathrm{Nu}-$ trition Examination Survey). O processo amostral foi por conglomerados e as análises bruta e ajustada (Regressão de Poisson) levaram em conta o efeito de delineamento. A prevalência de sobrepeso em adolescentes foi de 19,3\% (IC95\%: 16,6-22,0) e não houve diferença entre os sexos. Adolescentes pertencentes aos níveis econômicos mais elevados, que realizaram dieta para emagrecer nos três meses anteriores à entrevista, que assistiam quatro horas ou mais de televisão por dia e que realizavam apenas uma ou duas refeições diárias apresentaram maior proporção de sobrepeso. Na análise ajustada, o sobrepeso nos meninos esteve diretamente associado com nível econômico e, nas meninas, com as variáveis dieta para emagrecer, número de horas diárias assistindo à televisão e inversamente associado com o número de refeições.

Adolescente; Avaliação Nutricional; Sobrepeso 


\section{Colaboradores}

C. L. Dutra participou de todas as fases de produção do artigo, desde o planejamento até a coleta e análise dos dados e redação. C. L. Araújo contribuiu no planejamento, interpretação dos achados e na revisão crítica do artigo. A. D. Bertoldi participou do planejamento, análise dos dados e na revisão crítica do manuscrito.

\section{Referências}

1. Tojo-Sierra R, Leis-Trabazo R. La obesidad, un problema emergente en pediatría. Conferencia inaugural del VIII Congreso Nacional de la Sociedad Española de Nutrición, Murcia, 24-27 de octubre de 2001. Nutr Hosp 2002; 17:75-9.

2. Coitinho D, Leão M, Recine E, Sichieri R. Condições nutricionais da população brasileira: adultos e idosos. Brasília: Ministério da Saúde/Instituto Nacional de Alimentação e Nutrição; 1991.

3. Gigante DP, Barros FC, Post CL, Olinto MT. Prevalência de obesidade em adultos e seus fatores de risco. Rev Saúde Pública 1997; 31:236-46.

4. Troiano RP, Flegal KM. Overweight children and adolescents: description, epidemiology, and demographics. Pediatrics 1998; 101 (3 Pt 2):497-504.

5. Abrantes MM, Lamounier JA, Colosimo EA. Prevalência de sobrepeso e obesidade em crianças e adolescentes das regiões Sudeste e Nordeste. J Pediatr (Rio J) 2002; 78:335-40.

6. World Health Organization. Physical status: the use and interpretation of anthropometry. Report of a WHO Expert Committee. World Health Organ Tech Rep Ser 1995; 854:1-452.

7. Monteiro PO, Victora CG, Barros FC, Tomasi E. Diagnóstico de sobrepeso em adolescentes: estudo do desempenho de diferentes critérios para o Índice de Massa Corporal. Rev Saúde Pública 2000; 34:506-13.

8. Post CL, Victora CG, Barros FC, Horta BL, Guimarães PR. Desnutrição e obesidade infantis em duas coortes de base populacional no Sul do Brasil: tendências e diferenciais. Cad Saúde Pública 1996; 12 Suppl 1:49-57.

9. Gigante DP, Victora CG, Araujo CL, Barros FC. Tendências no perfil nutricional das crianças nascidas em 1993 em Pelotas, Rio Grande do Sul, Brasil: análises longitudinais. Cad Saúde Pública 2003; 19 Suppl 1:S141-7.

10. Gortmaker SL, Must A, Perrin JM, Sobol AM, Dietz WH. Social and economic consequences of overweight in adolescence and young adulthood. N Engl J Med 1993; 329:1008-12.

11. Rossner S. Obesity: the disease of the twenty-first century. Int J Obes Relat Metab Disord 2002; 26 Suppl 4:S2-4.

\section{Agradecimentos}

Esta pesquisa teve apoio financeiro da CAPES (Coordenação de Aperfeiçoamento de Pessoal de Nível Superior).

12. World Health Organization. Obesity: preventing and managing the global epidemic. Geneva: World Health Organization; 1998.

13. Monteiro CA, Mondini L, Souza AL, Popkin BM. The nutrition transition in Brazil. Eur J Clin Nutr 1995; 49:105-13.

14. Monteiro PO, Victora CG, Barros FC, Monteiro LM. Birth size, early childhood growth, and adolescent obesity in a Brazilian birth cohort. Int J Obes Relat Metab Disord 2003; 27:1274-82.

15. Parsons TJ, Power C, Logan S, Summerbell CD. Childhood predictors of adult obesity: a systematic review. Int J Obes Relat Metab Disord 1999; 23 Suppl 8:S1-107.

16. Troiano RP, Flegal KM, Kuczmarski RJ, Campbell SM, Johnson CL. Overweight prevalence and trends for children and adolescents. The National Health and Nutrition Examination Surveys, 1963 to 1991. Arch Pediatr Adolesc Med 1995; 149:1085-91.

17. Laitinen J, Power C, Jarvelin MR. Family social class, maternal body mass index, childhood body mass index, and age at menarche as predictors of adult obesity. Am J Clin Nutr 2001; 74:287-94.

18. Lohman T, Roche A, Martorell R. Anthropometric standardization reference manual. Champaign: Human Kinetics Books; 1988.

19. Altman DG. Practical statistics for medical research. London: Chapman \& Hall; 1991.

20. Barros AJ, Hirakata VN. Alternatives for logistic regression in cross-sectional studies: an empirical comparison of models that directly estimate the prevalence ratio. BMC Med Res Methodol 2003; $3: 21$

21. Victora CG, Huttly SR, Fuchs SC, Olinto MT. The role of conceptual frameworks in epidemiological analysis: a hierarchical approach. Int J Epidemiol 1997; 26:224-7.

22. Cole TJ, Bellizzi MC, Flegal KM, Dietz WH. Establishing a standard definition for child overweight and obesity worldwide: international survey. BMJ 2000; 320:1240-3.

23. Ogden CL, Flegal KM, Carroll MD, Johnson CL. Prevalence and trends in overweight among US children and adolescents, 1999-2000. JAMA 2002; 288:1728-32. 
24. Neutzling MB, Taddei JA, Rodrigues EM, Sigulem DM. Overweight and obesity in Brazilian adolescents. Int J Obes Relat Metab Disord 2000; 24:86974.

25. Veiga GV, Cunha AS, Sichieri R. Trends in overweight among adolescents living in the poorest and richest regions of Brazil. Am J Public Health 2004; 94:1544-8.

26. Kuczmarski RJ. Prevalence of overweight and weight gain in the United States. Am J Clin Nutr 1992; 55 (2 Suppl):495S-502S.

27. Danadian K, Lewy V, Janosky JJ, Arslanian S. Lipolysis in African-American children: is it a metabolic risk factor predisposing to obesity? J Clin Endocrinol Metab 2001; 86:3022-6.

28. Wang Y, Monteiro C, Popkin BM. Trends of obesity and underweight in older children and adolescents in the United States, Brazil, China, and Russia. Am J Clin Nutr 2002; 75:971-7.

29. Fonseca VM, Sichieri R, Veiga GV. Fatores associados à obesidade em adolescentes. Rev Saúde Pública 1998; 32:541-9.

30. Zimmermann MB, Hess SY, Hurrell RF. A national study of the prevalence of overweight and obesity in 6-12 y-old Swiss children: body mass index, body-weight perceptions and goals. Eur J Clin Nutr 2000; 54:568-72.
31. Silva RCR, Malina RM. Sobrepeso, atividade física e tempo de televisão entre adolescentes de Niterói, Rio de Janeiro, Brasil. Rev Bras Cienc Mov 2003; 11:63-6.

32. Hanley AJ, Harris SB, Gittelsohn J, Wolever TM, Saksvig B, Zinman B. Overweight among children and adolescents in a Native Canadian community: prevalence and associated factors. Am J Clin Nutr 2000; 71:693-700.

33. Robinson TN. Reducing children's television viewing to prevent obesity: a randomized controlled trial. JAMA 1999; 282:1561-7.

34. Strauss RS, Mir HM. Smoking and weight loss attempts in overweight and normal-weight adolescents. Int J Obes Relat Metab Disord 2001; 25:1381-5.

35. Malcon MC, Menezes AM, Chatkin M. Prevalência e fatores de risco para tabagismo em adolescentes. Rev Saúde Pública 2003; 37:1-7.

36. Frisancho AR. Prenatal compared with parental origins of adolescent fatness. Am J Clin Nutr 2000; 72:1186-90.

Recebido em 06/Dez/2004

Versão final reapresentada em 08/Abr/2005 Aprovado em 30/Mai/2005 